\title{
Crisis plebiscitaria de la democracia ecuatoriana
}

"En América Latina somos buenos para derrocar Presidentes. Deberíamos mostrarnos igualmente capaces para remplazarlos" (Evo Morales, Le Monde Diplomatique, Nov. 2005).

José Sánchez Parga*

Si en algo todo el mundo coincide hoy en el Ecuador es en reconocer la gravedad de la crisis que atraviesa el país ${ }^{1}$. Muchos en cambio son los desacuerdos en cuanto a la caracterización de dicha crisis y en la identificación de sus causas, aunque unos y otros vean por doquier sus culpables y responsables. Creer que de la crisis son responsables los que hoy actúan en ella, llevaría a suponer que son esos mismos actores, quienes podrían solucionarla. Por ello, la dificultad y el primer gran desafío, consiste en tipificar políticamente dicha crisis, entenderla a partir de las razones que la han provocado, a partir de los procesos de corta, pero también de mediana y larga duración, que mejor la contextualizan. Y esto precisando cuáles son los factores más directos o inmediatos que han desencadenado la crisis, cuáles son aquellas más estructurales y qué mediaciones entre ambos han reforzado tanto su continuidad como su actual agravamiento.

\footnotetext{
* Docente de la Universidad Politécnica Salesiana, investigador del Centro Andino de Acción Popular (CAAP), Quito.

${ }^{1}$ Todos en el país menos el Presidente de la República, Palacios, quien en rueda de prensa televisada el sábado 29 de octubre de 2005 declaró que "no hay ninguna crisis y todo lo que sucede es normal".
} 
En términos generales sería miope no advertir que la actual crisis socio-política es resultado extremo de una progresiva "desconsolidación" y "deslegitimación" de la democracia, la cual viene siendo observada desde hace casi una década ${ }^{2}$. De hecho el creciente poder plebiscitario, que ha emergido de la sociedad, se ha ido consolidando a medida que fuerzas e intereses oligárquicos han debilitado y deslegitimado los gobiernos e instituciones democráticos. Cuando las leyes e instituciones se desmoronan, surgen las fuerzas plebiscitarias en la sociedad conducidas por demagogos, "siendo el desenfreno de los demagogos la principal causa de las revoluciones en la democracia"3. En cualquier caso por plebiscitario habría que entender siempre fuerzas y poderes no constitucionales ni institucionales, y más bien opuestos a toda forma de constitucionalidad e institucionali$\mathrm{dad}^{4}$.

El "caos democrático" no es nuevo ${ }^{5}$. La novedad consiste en el más reciente descubrimiento de que la crisis es inherente a la misma democracia y a la contradicción que la funda: por un lado la imposibilidad de supeditar la domina-

${ }^{2}$ Cfr. SÁNCHEZ PARGA, José. Desconsolidación de la democracia y destrucción del sistema político, Caap. Quito, 2001; “¿Por qué se deslegitima la democracia? El desorden democrático", en Ecuador Debate, n. 62, agosto, 2004; “Quelle légitimité pour les démocraties andines?" en VVAA. Dépendences et démocratie en Amérique Latine, Colophon Editions, Bruxelles, 2004.

${ }^{3}$ ARISTÓTELES, Política, V, iii, 1304 b.

${ }^{4}$ Según GEMMA, Gladio, el calificativo de plebiscitario indicaría "sucesos excepcionales, normalmente fuera de las previsiones constitucionales... pronunciamientos populares no precedidos por actos estatales, y sobre todo hecho, o ya sea suceso (no actos normativos), que debido a su excepcionalidad no encontraría una disciplina constitucional": N. Bobbio et al., Diccionario de Política, Siglo XXI, México, 2000: 1183.

${ }^{5}$ TORRES-RIVAS, Edelberto. "El caos democrático", en Nueva Sociedad, n. 144, julio-agosto, 1996. Quizás en aquel entonces se pensaba todavía que la democracia podía salvarse del caos en el que se hallaba, pero ya después se entendió mejor que dicho caos era producto del mismo "desorden democrático". 
ción al gobierno y de eliminar el dominio por el gobierno democrático, cuando "gobernar ejerciendo una dominación es un error" y un horror democráticos 6 ; por otro lado, el hecho de ser el "menos malo" de todos los regímenes de gobierno atribuya a la democracia una legitimidad incondicional y una capacidad de legitimación ilimitada, incluso contra sus principios y presupuestos. Otra variante de la contradicción democrática es que un gobierno democrático solo se constituye con el apoyo del pueblo, pero solamente puede gobernar con el apoyo de las oligarquías.

\section{Morfología institucional de la crisis}

El fenómeno más visible de la crisis ha sido la pugna entre el Congreso y el Ejecutivo. Ahora bien la "pugna de poderes" entre ambos organismos del Estado no es nueva en la democracia ecuatoriana, ya que tiene sus raíces y presupuestos en el mismo modelo presidencialista del régimen democrático, pero se ha vuelto crónica del mismo sistema político, debido a un constante y renovado desequilibrio y tensiones entre las fuerzas parlamentarias de los partidos y los poderes presidenciales del Ejecutivo ${ }^{7}$. Pero mientras que hasta ahora los efectos de esta "pugna" se metabolizaban siempre por el resto del sistema político, por una suerte de metástasis conflictiva por todos los otros organismos del Estado, en la actual crisis dicha "pugna" arrastra también a la sociedad civil, para ser compartida por las mismas fuerzas sociales y populares.

Esta "pugna de poderes" se agrava aún más a raíz de la última Constitución de 1998, al quedar debilitados los poderes del Congreso (despojado, por ejemplo, de sus

\footnotetext{
${ }^{6}$ ARISTÓTELES. Política, VII, iii, 1325a.

${ }^{7}$ Cfr. SÁNCHEZ PARGA, José. La pugna de poderes. Análisis crítico del sistema político ecuatoriano, Abya-Yala, Quito, 1998.
} 
facultades de fiscalización y control del Ejecutivo), y al haber adquirido el Ejecutivo mayores poderes presidenciales (por las falaces razones de una mayor "gobernabilidad") ${ }^{8}$. Pero en contra de lo que se hubiera podido esperar, el fortalecimiento del Ejecutivo acarreó un peligroso debilitamiento de las funciones parlamentarias del Legislativo, lo que tuvo como consecuencia una perversión de la "pugna", una creciente corrupción de las relaciones entre diputados o partidos y el Ejecutivo y convertirse en una causa principal de la inestabilidad gubernamental.

Este error ha extremado la descomposición de las relaciones políticas no solo entre los poderes del Estado, sino también entre la creciente politización de la sociedad civil y sus relaciones con las otras instituciones democráticas: puesto que cuanto más fuerza acumula uno de los poderes más se desestabilizan los otros poderes. Esto explica, por ejemplo, que el poder de la calle o el poder de los mass-media solo haya podido fortalecerse a consecuencia del debilitamiento de las instituciones políticas; debilidad esta que aquellos poderes continuarán precarizando.

El desequilibrio de los "poderes en pugna" entre Congreso y Ejecutivo se encuentra a la base no sólo de una corrupción y deterioro de las relaciones entre ambos organismos del Estado, sino también han sido la causa de las inestabilidades gubernamentales de los últimos diez años, con el saldo de tres Presidentes de la República derrocados, uno de ellos en prisión junto con un ex-Presidente (Vicepresidente).

\footnotetext{
${ }^{8}$ En aquel entonces se cometieron dos graves errores: a) utilizar la Constitución para reparar errores políticos o ejercer un control político; b) desconocer que la "gobernabilidad" no se resolvía con mayor cantidad poder del Ejecutivo, lo que provocaría el reforzamiento de contrapoderes, sino con una mejor cualidad de poder, que mejorara las relaciones entre los poderes de la democracia.
} 
Pero lo más significativo de la crisis actual es que: a) en primer lugar, la "pugna" dos poderes extremadamente débiles, causa y consecuencia de un mutuo debilitamiento, y ambos Congreso y Ejecutivo con un bajísimo nivel de legitimación entre la opinión pública $(10 \%$ y $14 \%$ respectivamente); con bloques parlamentarios tan enfrentados entre ellos como divididos internamente y huérfanos de sus propios partidos y en fin con un Presidente políticamente minusválido, sin partido que lo apoye; b) en segundo lugar, a tal debilitamiento de los poderes institucionales ha correspondido un reforzamiento político de la sociedad civil y de poderes plebiscitarios a su interior ${ }^{9}$.

Es por consiguiente la fragilidad de los dos principales poderes del Estado y su profunda deselegitimación, lo que convierte su confrontación en una bomba de tiempo, que amenaza con desestabilizar todo el sistema político, sobre todo en el caso de que cualquiera de los dos recurra a medidas desesperadas o extremas: o bien si el Congreso entabla un juicio político para destituir al Presidente o bien si este intenta una clausura del Congreso. Sin llegar a tales desenlaces catastróficos de la crisis, los poderes políticos (partidos) y sobre todo el Presidente, podrían tratar de movilizar al pueblo a su favor, enrolándolo en la causa de una Asamblea Constituyente o Constitucional, que además de proporcionar apoyo a la gestión presidencial, le prestara un mínimo de legitimidad. Ahora bien, la Asamblea Constituyente supondría un desafío contra un Congreso, convertido por los "movimientos sociales" y las movilizaciones populares en cabeza de turco de la crisis del país y en el ejemplo

\footnotetext{
${ }^{9}$ Entendemos por plebiscitario ("plebe") el poder del pueblo, que en la antigua república romana se expresaba solo en las urnas ("plebiscitos") y que a través de sus representantes ("tribunos de la plebe" elegidos en "asambleas de la plebe"), solo ejercían un contra-poder, capaz de vetar leyes (del Senado) o limitar el gobierno del Ejecutivo (de los Cónsules).
} 
más representativo de la corrupción nacional. Mientras que reconocerle al Congreso la atribución constitucional de reformar la Constitución vigente, sería reforzar su poder y legitimidad, pero con ello el Presidente perdería la ocasión de reforzar y legitimar el Ejecutivo, y las fuerzas plebiscitarias perderían también su oportunidad para reforzarse y legitimarse políticamente.

Otra consecuencia de cualquiera de estas soluciones sería la desmovilización de las fuerzas y "movimientos sociales", organizaciones políticas de la sociedad civil y grupos agitacionales, que en la misma crisis han buscado su propia legitimidad política. El grito de "todos fuera", lanzado por las movilizaciones del abril del 2005, con el que se comienza derrocando al Presidente Gutiérrez y que servirá después para hostigar a los diputados e instituciones políticas, parece haberse convertido en el slogan y programa plebiscitario de todas las ulteriores movilizaciones sociales en el país.

Dos soluciones podrían visualizarse para evitar un desenlace catastrófico de la crisis. La primera consistiría en la búsqueda de un mediador nacional capaz de negociar o pactar una solución entre los poderes institucionales enfrentados. Pero la crisis ha alcanzado tal envergadura, que todas las demás crisis sociales de una u otra manera se encuentran confrontadas en ella, y sería muy difícil hallar un grupo que tomara una cierta distancia respecto del enfrentamiento generalizado, para desempeñar funciones mediadoras. Dentro de los movimientos sociales el movimiento indígena sería el único que, por su específico peso social y político y por su mayor legitimidad (de lo que carecen los otros movimientos sociales), gozaría de una relativa fuerza y autoridad. Sin embargo, no deja de ser sintomático que tal posibilidad de mediación no haya sido advertida por la opinión pública ni mucho menos por los mismos contendientes en el conflicto. 
Otra solución alternativa podría cifrarse en unas elecciones anticipadas (tampoco previstas constitucionalmente), que devolvieran una relativa legitimidad y fuerza social a los poderes políticos institucionales del Congreso y del Ejecutivo. Pero ello supondría que tanto los diputados como el Presidente abdicaran de sus actuales condiciones y pretensiones políticas. De otro lado esta posible solución no solo sorprende a los partidos políticos en un calamitoso estado de desmoronamiento, sino que además daría lugar a que la desesperación social propiciara la elección de las candidaturas más desesperadas. Finalmente, tampoco esta solución interesaría a quienes además de beneficiarse de la deslegitimación y debilitamiento de los poderes institucionales, pretenderían seguir sacando provecho político de la misma crisis ${ }^{10}$.

\section{La formación de un poder plebiscitario}

Por poder plebiscitario habría que entender aquellas fuerzas populares, que más allá de expresarse en las urnas y en sus propias formas de organización tienden a ejercerse sobre el conjunto de la sociedad, presionando a las mismas instituciones políticas y poderes del Estado. Este poder plebiscitario, que se ha ido fraguando en la sociedad ecuatoriana durante los últimos años, parece haberse convertido en uno de los factores de la crisis actual, llegando a formar

\footnotetext{
${ }^{10} \mathrm{Si}$ por un lado las FF.AA. se encuentran descalificadas para desempeñar una función mediadora, debido al horror constitucional, que las convierte en "garantes" del mismo orden constitucional, atribuyéndoles de hecho una equívoca y peligrosa función de "arbitraje", por otro lado, los negocios e intereses económicos de las FF.AA. han prosperado de tal manera, sobre todo desde la paz con el Perú, que no solo se mantienen al margen de las crisis nacionales, sino que además se aprovechan de ellas, al poder seguir negociando sus prebendas con poderes institucionales débiles y deslegitimados.
} 
parte de la correlación de fuerzas y de los conflictos que la caracterizan.

Que en América Latina los movimientos sociales fueron siempre más políticos que sociales (más "reactivos" que "proactivos" y "más teatrales que eficaces"), es una tesis muy documentada y ampliamente compartida ${ }^{11}$. Pero en el transcurso de la última década, por factores y razones que ya han sido analizados, el conflicto social ha dado lugar a una nueva forma de lucha: el ciclo político de la protesta, con el que los movimientos sociales se han ido transformando (aun sin saberlo ni quererlo) en movilizaciones sociales portadoras de una violencia protestaria, impugnadora y de rechazo contra los gobiernos y gobernantes, contra la clase política y contra las mismas instituciones democráticas ${ }^{12}$.

Esta politización de los movimientos sociales ha correspondido a una también creciente politización de la sociedad civil en su conjunto, la cual sobre todo en el transcurso de la última década se ha ido en parte privatizando y en parte politizando. La combinación de este doble fenómeno, movilizaciones de protesta y politización de la sociedad civil, ha constituido la principal fuerza de desestabilización del régimen democrático ecuatoriano durante los últimos 15 años, la cual ha provocado la destitución, expulsión o caída de los últimos tres presidentes constitucionales.

Lo nuevo de este proceso, y lo que caracterizaría de manera más específica la crisis actual, serían las formas de organización políticas, que han adoptado las movilizaciones, fuerzas y grupos sociales más diversos. Las protestas

${ }^{11}$ Cfr. TOURAINE, Alain. Actores sociales y sistemas políticos en América Latina, PREALC, Santiago, 1978; La Parole et le Sang. Politique et société en Amérique Latine, Odile Jacob, París, 1988.

${ }^{12}$ Cfr. SÁNCHEZ PARGA, José. "Transformaciones del conflicto, decline de los movimientos sociales y teoría del desgobierno", en Ecuador Debaten. 53, agosto, 2003; "Del conflicto social al ciclo político de la protesta", en Ecuador Debate, n. 64, abril, 2005.

234 
han dado lugar a posicionamientos, iniciativas y actividades políticas tendientes a constituirse en un real poder político al margen de toda institucionalidad, enfrentado con los otros poderes institucionales del Estado. Por primera vez se identifican "movimientos políticos" y "organizaciones políticas", habiéndose formado incluso organizaciones políticas con miembros o representantes de partidos ${ }^{13}$. La calle deja de ser un simple escenario social para las movilizaciones y las protestas y ha pasado a convertirse en un real campo político, desde donde se hace política; la calle ha dejado de ser un escenario político ocasional o extraordinario, para volverse recurrente y habitual; y sobre todo un poderoso instrumento de la agitación política de las fuerzas plebiscitarias ${ }^{14}$.

Es con el rechazo de toda representación e institución políticas, que las movilizaciones, fuerzas y grupos sociales se "constituyen" en un poder político plebiscitario, que paradójicamente se reivindicaría democrático, pero que se manifiesta opuesto a toda institucionalidad democrática; defiende los principios democráticos, en los que se funda, pero rechaza los procedimientos de la democracia y su gobierno. Cabe suponer que la formación de esta movilización y poder plebiscitarios en el régimen democrático ecuatoriano solo ha sido posible como resultado de la progresiva deslegitimación y debilitamiento de las instituciones democráticas y de la desinstitucionalización de la misma democracia. Pero hay que considerar en qué medida y con qué alcances la actual consolidación de este poder plebisci-

\footnotetext{
${ }^{13}$ Como es el caso de la agrupación de mujeres de seis partidos políticos de tendencias muy diferentes, o de agrupaciones políticas formadas por miembros de diferentes "movimientos sociales" y partidos. Ecuador se encontraría en el umbral de sustituir el nombre de partido por el de agrupaciones políticas, que tendrían la misma función política y estarían integrados por los mismos políticos, como ha sido el caso en Bolivia.

${ }^{14}$ Cfr. CIZEK, Eugen. Mentalités et institutions politiques romaines, Fayard, Paris, 1990.
} 
tario (en oposición a la "desconsolidación de la democracia"), puede extremar aún más hasta umbrales catastróficos el quebrantamiento institucional de la democracia. Cuando el pueblo insatisfecho con los resultados de su ejercicio de poder en las urnas, pretende imponerlo en las mismas instituciones democráticas, legislativas y gubernamentales. El poder del pueblo fue siempre importante y necesario tanto para la agitación política como para la subversión de los poderes establecidos, pero no en cuanto ejercicio de gobierno. Es entonces, que el mismo pueblo se vuelve despótico ${ }^{15}$.

En este sentido, y más allá de la aparente paradoja de los llamados "movimientos sociales" y las movilizaciones de grupos y organizaciones más diversos de la sociedad civil (desde ONGs hasta organismos de Derechos Humanos) hayan cifrado su actual lucha en una Asamblea Constituyente, la solución de la crisis en una nueva Constitución no tendría más finalidad, en primer lugar, que legitimarse políticamente, y en segundo lugar, hacer de una nueva Constitución no un sistema de principios republicanos y democráticos, sobre el cual se fundaría toda posible legislación y gobierno, sino un instrumento de reivindicaciones concretas (desde el rechazo del TLC, de la base de Manta o la explotación petrolera extranjera), sino también un arma contra la misma clase política.

Por eso, estos furores plebiscitarios y constitucionalistas de muchos grupos y de los mismos movimientos sociales han de entenderse además porque su organización y posicionamientos políticos les plantean ante sí mismos y ante la opinión pública un problema inédito de legitimidad.

\footnotetext{
${ }^{15}$ Esta situación ha sido descrita ya por Aristóteles, "cuando las masas y no las instituciones o leyes son soberanas"; siendo entonces "cuando las leyes o instituciones no son soberanas, que emergen los demagogos", y de esta manera "el mismo pueblo se convierte en déspota" (Aristóteles, Política, IV, iv, 1292b).
} 
Pues mientras que actuaban como "movimientos sociales" el criterio de su legitimidad se fundaba en su poder de convocatoria y de conducción de sus bases, cuando se presentan con actuaciones y posiciones políticas la cuestión de su legitimidad se plantea de otra manera: ¿a quiénes representan? ¿quién los ha elegido? ¿Cómo fueron elegidos? Estas cuestiones constituyen el auténtico núcleo de los factores que atizan y envenenan la crisis: el conflicto de legitimidades tanto entre los poderes instituidos como entre estos y las fuerzas plebiscitarias.

Según esto bajo la diversa morfología adoptada por la crisis actual, lo que parece caracterizarla de manera muy específica es su naturaleza plebiscitaria: la que enfrenta al pueblo no solo contra el gobierno, la clase política, el Presidente y Ejecutivo o el Congreso, sino contra toda la institucionalidad democrática y contra todo poder político institucionalizado, atribuyendo al pueblo capacidades o competencias gobernantes. No es por eso casual que la actual crisis haya estado preparada y ambientada por una ideología y un discurso participacionistas, que ante el fracaso de la democracia representativa reivindiquen una democracia participativa o democracia directa. Aunque pocos se hayan interrogado sobre cómo entender tal participación y cómo funcionaría ${ }^{16}$.

Las fuerzas y movilizaciones plebiscitarias pasaron de tener articulaciones más institucionales, primero aliadas a fuerzas y partidos políticos, para irse independizando cada vez más de ellos, alcanzando una autonomía que reforzaba su doble carácter anti-institucional y popular. Así, mientras que en el primer derrocamiento presidencial de Bucaram (1996) los sectores populares de protesta y los

\footnotetext{
${ }^{16}$ Un muy elemental análisis político demostraría fácilmente que las razones por las cuales ha fracasado la democracia representativa son las mismas que harían inviable una democracia participativa.
} 
movimientos sociales se movilizaron junto con partidos políticos desde el Congreso nacional, en el segundo derrocamiento presidencial (2000), el de Mahuad, las movilizaciones populares y los movimientos sociales (conducidos por el movimiento indígena) junto con militares golpistas (entre ellos el actual Alcalde de Quito y el que sería futuro Presidente, coronel Lucio Gutiérrez), primero asaltan y se toman el Congreso, para después tomarse el Palacio presidencial; en el tercer derrocamiento presidencial de Gutiérrez las movilizaciones populares se forman en las calles no solo al margen de los partidos o fuerzas políticas sino incluso al margen de los mismos movimientos sociales, ausentes en los episodios definitivos del 20 de abril del 2005.

Este progresivo fortalecimiento de las movilizaciones plebiscitarias y de su poder político, que alcanza su fase culminante el 20 de abril, no concluye como en las anteriores con el derrocamiento del Presidente de la República sino que al grito de "todos fuera" pretende también la clausura del Congreso y la revocación del mandato de los diputados. A diferencia de los anteriores episodios de desestabilización democrática (presidencial), aunque por efecto de su acumulación, el último se caracterizará por el intento de desestabilizar también el Congreso nacional y de clausurarlo, lo que en aquel momento hubiera supuesto el completo desmoronamiento del régimen democrático (sin Ejecutivo, sin Legislativo, sin Corte Suprema de Justicia y sin Tribunal Constitucional).

Además de ello, lejos de pasar a una fase de tregua o receso, como período de diástole normal tras las grandes crispaciones políticas, las fuerzas y movilizaciones populares entraron en un proceso de organización política junto con sectores y grupos muy diversos de la sociedad, desde ONGs hasta medios de comunicación, manteniendo un constante acoso y hostigamiento al Congreso, a los diputados y al nuevo Ejecutivo; cifrando en el proyecto de una 
Asamblea Constituyente no solo todas las desesperaciones populares, todas las esperanzas de cambio y la única posible resolución de la crisis, sino también la oportunidad para el fortalecimiento político de los líderes y organizaciones populares.

Este nuevo fenómeno plebiscitario tiene sus raíces en la particular conformación de las fuerzas movilizadas durante el golpe de abril, las cuales además de no haber sido conducidas por partidos, ni por los movimientos sociales ni por otras organizaciones de la sociedad civil, fueron sociológicamente tan heterogéneas y tan anónimas, que terminaron por identificarse con el calificativo de "foragidos", acusación lanzada por el gobierno contra los agitadores de una radiodifusora de Quito, y que después sería muy ampliamente adoptada por los más diversos sectores de la población como distintivo de su oposición al gobierno ${ }^{17}$. Lo cual demuestra cómo las fuerzas y discursos plebiscitarios han llegado a permear los más diferentes sectores, grupos y clases de la sociedad y cómo sus dinámicas latentes son capaces de resurgir y movilizarse en cualquier circunstancia.

\section{Reacción plebiscitaria a la crisis global de democracia}

Hasta ahora se había advertido y enfatizado de manera casi exclusiva uno de los factores principales de la crisis de la democracia moderna en su tendencia autoritarista, dominada por fuerzas e intereses económicos del nuevo orden global. $Y$ en este sentido recientemente se ha destacado con frecuencia que "la dictadura es la forma de gobier-

\footnotetext{
${ }^{17}$ Radio La Luna, gran protagonista de la protesta y agitación que terminó con el derrocamiento de Gutiérrez, había hecho una campaña militante tres años antes a favor de la candidatura presidencial del mismo Crln. Gutiérrez. En septiembre del 2005 el Municipio de Quito organiza una mesa redonda para tratar la quiteñidad de las movilizaciones que derrocaron al Presidente Gutiérrez. Hay que recordar, sin embargo, que el 74.8\% de los quiteños habían votado por el Crnl. Gutiérrez para la Presidencia de la Republica menos de dos años antes.
} 
no del imperio", al que hoy se encuentran sometidas todas las democracias del mundo ${ }^{18}$. Hace casi quince años, se podía advertir ya "el totalitarismo neoliberal", que podría amenazar con destruir la democracia o al menos su eficacia y legitimidad políticas ${ }^{19}$. Sin embargo se ha desconocido o relegado el otro factor, que puede poner en crisis la democracia, llegando a desestabilizarla de manera tan extrema como irreversible: las fuerzas y movilizaciones populares, capaces de imponerse sobre las instituciones y gobierno democráticos, para terminar con su destrucción. No se puede obviamente desvincular esta crisis plebiscitaria de la otra crisis totalitaria, que amenaza la democracia, puesto que aquella se manifiesta, refuerza y legitima en cuanto reacción contra ésta. Cuando las fuerzas e intereses oligárquicos dominan el gobierno republicano o democrático, y se imponen sobre sus instituciones, "harta la multitud de este gobierno" termina por rebelarse ${ }^{20}$. Basta una circunstancia coyuntural, un detonante, para que el vasto torrente contestatario, más o menos represado, "en períodos de tensión se transforme en fuerza y en acciones colectivas, que en el momento más agudo de la confrontación se articulen en movimientos de masas" ${ }^{\prime 1}$.

${ }^{18}$ PAYE, Jean-Claude. "La dictadure, forme de gouvernement de 1'Empire", en La Pensée, n. 340, 2004. El último voluminoso número de la Revista del MAUSS está precisamente dedicado al tema del Malestar en la democracia y el espectro del totalitarismo (Malaise dnas la Décmoratie. Le spectre du totalitarisme, La Découverte, n. 25, 2005.).

${ }^{19}$ Cfr. SÁNCHEZ PARGA, José. "Ecuador en el engranaje neoliberal”, en Nueva Sociedad, n. 123, enero-febrero, 1993.

20 "infastidita da'loro governi, la moltitudine..." (N. Maquiavelo, Discorsi sopra la Prima Deca di Tito Livio, I, 2. Tutte le opere, Edit. Sansoni, Firenze, 1992

${ }^{21}$ Estas declaraciones de García Linera, ideólogo del MAS de Evo Morales, que define el Movimiento al Socialismo más como una confederación de organizaciones sociales que como partido político, muestran la analogía entre la crisis boliviana y la ecuatoriana; aunque aquella ha dotado a sus fuerzas y condiciones prerrevolucionarias de una institucionalidad y programa políticos. 
El problema político que hoy se plantea, es que si bien la democracia es un régimen de gobierno (el "menos malo") tan ilimitadamente perfectible como ilimitadamente corruptible, capaz de soportar la peor de las dominaciones totalitarias, o bien se desmorona bajo las luchas plebiscitarias, o bien estas provocan su propia represión con la más extrema violencia. Y si la crisis plebiscitaria (el stato popolare, según Maquiavelo) es producto de una violenta reacción por parte del pueblo y amplios sectores de la sociedad contra el modelo de dominaciónexclusión ejercido a través del mismo régimen democrático y de sus instituciones, dicha crisis a su vez podría desencadenar un espiral de violencia política por parte de las fuerzas que dominan y se ejercen a través de los gobiernos e instituciones democráticos.

En este sentido, la actual crisis plebiscitaria del Ecuador es la eclosión de una crisis global de la democracia, que en todo el mundo da muestras de una creciente distancia y progresivo alejamiento entre los ciudadanos, el pueblo, y sus gobiernos y gobernantes; una brecha que no cesa de ensancharse entre los ciudadanos y sus diputados elegidos, cuya representación política cada vez representa menos a sus representados. Pero mientras que en Europa, EE.UU. y otros países desarrollados y de larga tradición democrática este creciente apartamiento de los gobiernos democráticos del pueblo de ciudadanos se halla relativamente atenuado al menos por el momento- por la mediación de sus instituciones sociales y democráticas, en Ecuador como en la mayor parte de países latinoamericanos las instituciones democráticas son parte del distanciamiento y ahondan aún más el abismo entre la democracia gobernante con 
su clase política y la democracia gobernada del pueblo ${ }^{22}$. Aunque las comparaciones deban ser utilizadas con sumo esmero analítico, habría que reconocer que la clase política ecuatoriana se muestra una de la más distante y divorciada de su propia sociedad ${ }^{23}$.

En este sentido las fuerzas y movilizaciones plebiscitarias, que caracterizan la actual crisis ecuatoriana no hacen más que "atacar el tabú de la democracia" (J. Saramago), y en nombre de los mismos principios democráticos impugnar y rechazar todo un ordenamiento y funcionamiento de las instituciones democráticas, comenzando por sus gobiernos, ya que constantemente contradicen y atentan contra dichos presupuestos de la democracia. Sin embargo, sin disculpar las responsabilidades de una clase política, cada vez menos política y más profesional, cada vez más representativa de sus orígenes e intereses de clase, y sin ignorar el síndrome de precariedad, corrupción y desesperación de los nuevos gobiernos y gobernantes democráticos, es preciso reconocer que las democracias actuales se encuentran secuestra-

${ }^{22}$ Una alternancia crónica ente gobiernos y oposición o bien la situación contraria de regímenes neoliberales que se perpetúan protegidos por el nuevo orden global, o bien el no rotundo de los ciudadanos contra consultas de una casta de "decidores" que toman decisiones nefatas contra la mayoría de los ciudadanos, todos estos y otros muchos fenómenos demuestran el actual horror democrático, que por fin comienza a ser cuestionado por los mismos demócratas. Cfr. Manieres de voir, n. 83, octubre - noviembre, 2005.

${ }^{23}$ Mientras que el $67 \%$ de los diputados ecuatorianos procede de la clase media/alta y alta, en comparación con el $40 \%$ del total latinoamericano, y el 66\% reconoce percibir más de 40 mil dólares de ingresos anuales, en comparación con el $63 \%$ del total latinoamericano, sin embargo el $45 \%$ de los diputados ecuatorianos percibe como "modesta" su condición socio-económica en comparación con el 35\% latinoamericano. Cfr. Elites parlamentarias Iberoamericanas: Ecuador, Univ. Salamanca /AECI / Inst. Investigaciones Sociológicas, Madrid, 1988. 
das, más o menos dominadas, condicionadas o constreñidas, por las fuerzas e intereses del nuevo orden mundial; de tal manera que son esta dependencia y sometimiento externos los que los separan y distancian de sus ciudadanos e incluso enfrentan con ellos.

Una comprensión de la naturaleza de la crisis actual de la democracia debe considerarla sometida y dominada por un totalitarismo no metropolitano, ejercido exteriormente desde un determinado centro geográfico, sino por un totalitarismo sistémico y global, que la penetra y la violenta internamente, ya que la voluntad de poder del totalitarismo sistémico no se objetiva sin la necesaria volunta de los mismos sujetos; y aunque a nivel geopolítico manifieste una cierta centralidad norteamericana, sus violencias y lógicas expansivas consisten en una progresiva ilimitación e internalización por las mismas instituciones y gobiernos de la democracia. La violencia sistémica no se presenta como un ejercicio de dominación, sino como una lógica y necesidad operatoria, función del orden, "un campo de fuerza gravitario generalidado", donde la destrucción y la muerte no son más que "momentos de condensación o "daños colaterales $^{24 "}$.

La democracia en todo el mundo se disuelve política, social y simbólica o valorativamente por efecto de otra forma política, social y simbólica no opuesta a ella sino producto de ella y generada por la misma democracia: el totalitarismo. Pero de nuevo hay que insistir en ello: los efectos devastadores del totalitarismo se distribuyen de manera muy desigual por el mundo y por sus democracias: en países desarrollados con democracias de larga consolidación

${ }^{24}$ Cfr. FREITAG, Miche. "Totalitarismes: de la terreur au meilleur des mondes”, en Revue du MAUSS, n. 25, La Découverte, 2005. 
encuentra sus límites; pero, por el contrario, sin una democracia social o sociedad democrática, la democracia política sería capaz de legitimar nuevas formas de dominación y de servidumbre más o menos "reguladas, suaves y apacibles" (Tocqueville) ${ }^{25}$.

El grado terminal y también más contradictorio de esta crisis plebiscitaria es que el radical rechazo por parte del pueblo de las instituciones democráticas haya tenido el efecto de distanciar a este mismo pueblos de su propia realidad, y de sus condiciones socio-política, haciéndole creer y cifrar desesperadamente todas sus esperanzas en una Asamblea constituyen y la solución de todos sus problemas en una nueva Constitución. Cuando de hecho estarían dadas todas las circunstancias, peores que las que tuvo la Constitución de 1998, para garantizar otra Constitución más contingente y más controversial: a) el nivel de crisis del país presenta un marco más adverso, aunque resulte muy sintomático y nada casual, que sean dos gobiernos de transición, carentes de legitimidad y sin fuerzas políticas los que en aquel entonces y ahora convocan a una Asamblea Constituyente; b) lejos de haberse propiciado una convocatoria de consensos amplios y diversos, se ha planteado en términos de exclusiones y descalificaciones anticonstitucionales; c) se pretende hacer de una nueva Constitución no tanto un instrumento constituyente de la realidad socio-política e institucional del país, cuando un instrumento político, que además de producir una realidad inexistente, se traduzca en normas y leyes y regulaciones, cuando la crisis actual radica precisamente en la deslegitimación de los fundamentos legales y normativos de todas las instituciones. Empezando

\footnotetext{
25 "Si el despotismo llegara a establecerse en las naciones democráticas actuales, tendría otras características; sería más extenso y más suave y degradaría a los hombres sin atormentarlos" (A. De Tocqueville, De la Démocratie en Amérique, t. II, p. 232, Gallimard, París, 1961).
} 
por la Constitución. Según esto, habría que suponer que la actual forma plebiscitaria de la crisis de democracia, es de tal envergadura, que las únicas solidas que en ella o desde ella se visualizan, lejos de resolverla podría o tendería más bien agravarla.

Si lo que se ha caracterizado como crisis plebiscitaria no es más que una forma extrema de la crisis de la democracia ecuatoriana, y si tal crisis no es más que una variación, parte y expresión de "la crisis de la democracia occidental", o mejor dicho, de la crisis de la democracia globalizada en todo el mundo, habría que preguntarse hasta qué punto es tratando sus síntomas o morfologías, que se pueden intervenir en las razones y causas más estructurales de la crisis. Pero más que resolver la crisis de la democracia y salvar a esta de su crisis, es preciso reconocer que tal crisis emerge de la profunda contradicción tan intrínseca como constitutiva de la misma democracia: siendo el "menos malo" de todos los regímenes políticos, puede ser tan ilimitadamente perfectible como ilimitadamente pervertible y corruptible. Precisamente esta ilimitada capacidad de degradación, la que le impide "tocar fondo", convierte la democracia en el más peligroso régimen político y de gobierno, y le permite resistir y soportar tanto las peores violencias ejercidas a través de sus gobernantes y de sus instituciones cuanto las más destructivas subversiones populares. Nadie como Maquiavelo entendió la violencia inherente al orden/desorden democrático: "De todas las violentas servidumbres, la más violenta es la que te somete a una democracia (república): en primer lugar, porque es la más duradera y no se puede salir de ella; en segundo lugar, porque es el fin de la democracia enervar y debilitar todos los otros cuerpos (del Estado), para fortalecer el propio ${ }^{26 "}$.

${ }^{26}$ MAQUIAVELO, Nicolas. Discorsi, II, 2. 
